\title{
Studying Kinetics by Counting Particles in Time-Resolved Cryo-EM
}

\author{
Joachim Frank ${ }^{1,2}$
}

${ }^{1}$ Department of Biochemistry and Molecular Biophysics, Columbia University, New York, USA

${ }^{2}$ Department of Biological Sciences, Columbia University, New York, USA

In time-resolved cryo-EM, the progress of a reaction, started by mixing of two components, can be followed over a period of time by stopping the reaction and imaging the samples at defined intervals [1, 2]. At each time point there will be a mixture of the two starting components, transient intermediate products, and end product. Most reactions of biological molecular machines of interest are completed within a few hundred milliseconds, necessitating special technology for fast mixing, reacting over a defined period of time, and depositing the reaction product on the EM grid. We have demonstrated that with a mixing/spraying microfluidic device previously developed in a collaboration between researchers at the Wadsworth Center and RPI [3], high-resolution reconstructions (in the 3-Å range) of short-lived intermediates can now be routinely obtained $[4,5]$. Thus, we can now achieve both spatial and temporal resolution in following the progress of a reaction. Importantly, kinetic data are obtained implicitly as a byproduct of classification: by simply counting the number of particles in each class (or subpopulation) for each time point captured. In principle, therefore, time-resolved cryo-EM is able to measure the kinetics of a macromolecular reaction by a sequence of experiments with microfluidic chips designed for a series of time points.

Here I would like to raise the question to what extent this method of kinetic measurement can be used in practice. This is an important question since a reliable method of measuring reaction kinetics of macromolecules by counting particles in micrographs collected by cryo-EM would be a significant addition to the tool chest of Biophysics.

However, the precise quantification of the numbers of particles in such experiments faces several obstacles: there is evidence that a fraction of molecules suffer damage as they are prepared for cryo-EM. This damage is attributed to denaturing interactions with the air-water interface [6], where the majority of the molecules reside according to recent electron tomographic evidence [7]. Even if there were a reliable way of sorting out damaged from undamaged molecules, then a bias in counting could still be created if the propensity to damage is different for different reaction products. Clearly a remedy to this problem would require a different preparation method that ensures all molecules are fully encased in ice. Next is the difficulty of unbiased particle selection. At the particle-picking step, it is possible that different molecule species are picked with different error rates. This problem requires a systematic study of particlepicking algorithms using mixtures of different species with defined ratios. Third on the list is the question if the classification algorithm is able to make the right decision in sorting the images into a pre-given number of classes, especially in the presence of "noise images", or false positives in the particle picking. Here it is useful to do tests on a given dataset with different fractions of fake particles (i.e., simulated false positives of particle picking) admixed, taking for as fake particles, for example, data excised from the background [4]. Fourth is the reproducibility of classification results from the same dataset with different seeds, which for a dataset of ribosome particles turned out extremely good, within a very small margin of error. 
In all, these questions define an entire program of validation tests for different kinds of molecules. These tests do not necessarily all require time-resolved experiments, but can be parceled into separate studies all involving conventional cryo-EM [8].

References:

[1] B Chen and J Frank, Microscopy 65 (2015), p. 69.

[2] J Frank, J. Struct. Biol. 200 (2017), p. 303.

[3] Z Lu, J. Struct. Biol. 168 (2009), p. 388.

[4] Z Fu, Nature Communications, in press (2019).

[5] S Kaledhonkar, Nature, in press (2019).

[6] AJ Noble, eLife (2018), https://doi.org/10.7554/eLife.34257.001.

[7] RM Glaeser and B -G Han, Biophys. Rep. 3 (2017), p. 1.

[8] This work was supported by NIH R01 GM55440. 